\title{
AUTORES Y TEXTOS TEATRALES ELECTRÓNICOS (EN CASTELLANO) DEL TEATRO ESPAÑOL CONTEMPORÁNEO
}

\author{
PLAYWRIGHTS AND ELECTRONIC THEATRICAL TEXTS \\ (IN CASTILIAN LANGUAGE) OF THE CONTEMPORARY \\ SPANISH THEATRE
}

\author{
Ricardo DE LA TORRE RODRÍGUEZ \\ Grupo de Investigación del SELITEN@T \\ ricardotorrerodriguez@gmail.com
}

Resumen: En el marco de la relación entre teatro y las nuevas tecnologías, una de las líneas de investigación llevadas a cabo en el Centro de Investigación de Semiótica Literaria, Teatral y Nuevas Tecnologías (SELITEN@T: http://www.uned.es/centro-investigacion-SELITEN@T/), el propósito de este artículo es realizar una aproximación a los autores del teatro español contemporáneo y a los textos completos en versión electrónica de sus obras (en castellano), disponibles para la lectura y descarga gratuita desde las páginas en red de los propios autores. Otras páginas seleccionadas completan y proporcionan al investigador o al lector de teatro una visión general de estos autores con los títulos de sus producciones, ordenados alfabéticamente. 
Abstract: On the relation between theatre and new technologies, one of the lines of research carried out at the Research Center of Literary and Theatrical Semiotics and New Technologies (SELITEN@T: http://www. uned.es/centro-investigacion-SELITEN@T/), the purpose of this article is to present an approach to the contemporary Spanish playwrights and the full-text electronic version of their plays (in Spanish), available for free reading and downloading from the own authors' websites. Some other selected sites also complete and provide the research worker or just the play reader with an overview of these authors with the titles of their productions, listed alphabetically.

Palabras clave: Autores. Siglo XXI. Textos electrónicos. Teatro español. Nuevas Tecnologías.

Key words: Authors. $21^{\text {st }}$ Century. Electronic texts. Spanish Theatre. New Technologies.

\section{INTRODUCCIÓN}

De las investigaciones realizadas en el Centro de Investigación de Semiótica Literaria, Teatral y Nuevas Tecnologías de la UNED, dirigido por el profesor José Romera Castillo, buena parte de ellas dan cuenta de la conexión y relación del teatro y las nuevas tecnologías. Para una muestra de lo investigado en este campo, del que es pionero el SELITEN@T, puede consultarse la reciente presentación trazada por su director José Romera Castillo (2013: 11-32), «Sobre Teatro e Internet en el SELITEN@ @ », en el vigésimo segundo Seminario Internacional, sobre Teatro e Internet en la primera década del siglo XXI, que tuvo lugar en la sede la UNED de Madrid, en colaboración con el Centro de Documentación Teatral (INAEM del Ministerio de Educación, Cultura y Deportes) y del Instituto del Teatro de Madrid, del 25 al 27 de junio de 2012 (Romera Castillo, ed., 2013). También en la citada publicación, el panorama «Internet e investigación teatral. Fuentes en la red para la investigación del teatro español de los siglos XIX y XX», de Berta Muñoz (2013: 33-60). Sobre otros Seminarios Internacionales anteriores dedicados al tema y organizados por el SELITEN@T: el sexto, que se realizó en la Universidad Internacional Menéndez Pelayo de Cuenca, del 1 al 4 de julio de 1996, fue publicado por José Romera Castillo, et alii (eds.), Literatura y multimedia (1997). El decimotercero, sobre Teatro, prensa y nuevas tecnologías (1990-2003) (Romera Castillo, ed. 2004), se celebró conjuntamente en la UNED y Casa de América (Madrid), del 25 
al 27 de junio de 2003. Para una ampliación sobre el tema véanse también, entre otras publicaciones del profesor José Romera, «Hacia un estado de la cuestión sobre teatro y nuevas tecnologías en España», en la revista Signa 17 (2008: 17-28), con «Presentación» de Dolores Romero (2008: 13-16) ${ }^{1}$; «Literatura, teatro y nuevas tecnologías: investigaciones en el SELITEN@T (España)», en Epos XXVI (2010: 409-420). Además de los capítulos de sus libros: «Sobre teatro y nuevas tecnologías», en Teatro español entre dos siglos a examen (Romera Castillo, 2011a: 388-409), así como el dedicado a «El Centro de Investigación de Semiótica Literaria, Teatral y Nuevas Tecnologías», en Pautas para la investigación del teatro español y sus puestas en escena (Romera Castillo, 2011b: 21-45).

Se sabe que el teatro es un arte diferente a la literatura con el que comparte el texto escrito y también que no existe en España una fuerte tradición en la publicación de estos textos teatrales (Fernando Olaya, 2013: 140-141). Dentro de este marco, la presente investigación se plantea, a modo de inventario, realizar una breve guía - que no completa relación- de los autores con sus textos teatrales en formato electrónico. Los listados de autores y títulos - que pueden leerse o descargarse gratuitamente a texto completoaparecen recogidos en los epígrafes dos y tres. Son puestos a disposición de los investigadores e internautas interesados, con las ventajas que posibilitan estas ediciones. Se han considerado los siguientes criterios y fuentes.

\subsection{Criterios}

- Para confeccionar esta guía de autores españoles de teatro, vivos o fallecidos en este siglo, con textos completos en línea, se ha tomado como punto de partida el directorio de la Asociación de Autores de Teatro. El listado (con fecha de última actualización a 10-3-10) abarca asociados a la entidad de todo el ámbito nacional y puede consultarse en la pestaña autores de la sección Quiénes somos (http://www.aat.es/index_quien.html). A los nombres, ahí registrados, se suman aquellos que en la propia investigación nos hemos encontrado. No por ello está la nómina cerrada, en todo caso, es un primer paso para la catalogación de este teatro en castellano, como podrá examinarse en el propio trabajo, ya que algunas de las fuentes y nombres

${ }^{1}$ En el mismo número de Signa, en el artículo «Teatro y nuevas tecnologías: conceptos básicos», Anxo Abuín (2008: 29-56), al tiempo que pasa revista a los conceptos básicos resultado de la relación entre teatro y nuevas tecnologías y comenta los resultados más relevantes, realiza una crítica a algunos modelos imperantes. 
abren a su vez puertas a futuras investigaciones con nuevas aportaciones e incrementos de autoría y títulos.

- Del listado de asociados a la AAT, en torno a unos trescientos — -según leemos en la presentación de la entidad-, se han considerado las autoras y los autores que cuentan con página electrónica personal. Berta Muñoz (2011: 217-218), en descripción detallada, señala cómo este portal de la Asociación de Autores de Teatro cuenta con varios cientos de obras teatrales de más de medio centenar de dramaturgos españoles. Su catálogo dispone de un índice alfabético de autores y otro de títulos digitalizados, entre los que se encuentran los de los textos teatrales. El número de autores que se incluyen en la AAT se reduce significativamente cuando se considera que cuenten con página electrónica personal; criterio establecido para los autores del epígrafe dos de esta investigación. Se han incluido en dicho epígrafe, de los textos teatrales en las páginas electrónicas de los autores, la colección de obras de las páginas de la biblioteca de autores. Estas páginas aparecen con la anotación AAT. Fruto del convenio de la Biblioteca Virtual Miguel de Cervantes y la Asociación de Autores de Teatro con el objetivo de dar a conocer destacadas obras del teatro español contemporáneo mediante ediciones digitalizadas, el listado de obras que se aloja en el citado portal se amplía y - sin dar cuenta aquí de todos los títulos por razones de espacio y de acotación de la investigación - sin embargo, como una fuente más, se tiene en cuenta para completar la información sobre los textos en red de otros autores. En este caso, aparecen anotados como CV.

- Se han listado los nombres alfabéticamente, con indicación del lugar y fecha de nacimiento, siempre que ha sido posible, seguido de la página o páginas electrónicas de la autora o autor; y a continuación los títulos de los textos teatrales, también alfabéticamente, con las abreviaturas, anotaciones, siglas y otros códigos que se detallan en el apartado correspondiente, según sea el caso. Estos autores y los títulos de sus obras teatrales aparecen recogidos en el epígrafe dos.

- A los títulos del epígrafe dos, concerniente a los textos teatrales en las páginas electrónicas de los autores, se ha optado por sumar los títulos hallados de la autora o autor en otras páginas - con fuentes entre paréntesispara no repetir los nombres en el epígrafe tres.

- El trabajo se completa en el epígrafe tres con aquellos otros autores que no cuentan con espacio propio en la Red, ya sea página personal o web en la biblioteca de autor de la AAT — considerados en el epígrafe dos-, pero representativos del teatro contemporáneo y a cuyos textos teatrales 
podemos acceder desde las fuentes que se indican. En este caso, no se recogen los títulos por las razones ya señaladas de espacio y de acotación de la propia investigación.

\subsection{Fuentes}

A las fuentes, ya aludidas, del catálogo de la Asociación de Autores Teatro y de las páginas de autor de estos, se suman la información aportada por el Archivo Virtual de las Artes Escénicas, Biblioteca Virtual Miguel de Cervantes, Centro de Documentación Teatral, Instituto de Tecnologías Educativas, Muestra de Teatro Español de Autores Contemporáneos y Parnaseo. Fuentes que permiten ampliar los títulos recogidos en el epígrafe dos, a la vez que aportan y permiten considerar nuevos nombres para la nómina de autores con textos en la Red del epígrafe tres.

\section{Archivo Virtual de las Artes Escénicas}

(http://artesescenicas.uclm.es)

Entre los materiales documentales de la mediateca más vanguardista del teatro en español, los del Archivo Virtual de Artes Escénicas, del grupo de investigadores ARTEA — una asociación independiente vinculada a través de sus miembros a diferentes universidades y centros de investigación-, se ponen a disposición textos teatrales de creadores escénicos contemporáneos iberoamericanos. En la sección dedicada a los textos teatrales en una tipología diversa — que abarca estudios, entrevistas o los textos propiamente dramáticos-, a fecha de julio de 2013, ofrece trescientos cincuenta y cinco registros; de estas creaciones dramáticas pueden leerse a texto completo, entre otros, las de los autores Roger Bernat, Marta Galán, Carlos Marquerie y Angélica Liddell.

\section{Biblioteca Virtual Miguel de Cervantes}

\section{(http://www.cervantesvirtual.com)}

Además de la colección de textos teatrales de la biblioteca autor de la Asociación de Autores de Teatro - anotado AAT - y fruto del acuerdo de la Biblioteca Virtual Miguel de Cervantes y la Asociación de Autores de Teatro, señalado en el apartado anterior, se recogen, a partir de las fichas de autores del buscador de la Biblioteca Virtual, otros títulos teatrales - anotado $\mathrm{CV}$ - Entre los varios cientos de documentos de teatro de muy diversa tipología (archivos sonoros, entrevistas, vídeos, imágenes, estudios, tesis...), 
destacan varios cientos de obras teatrales digitalizadas a texto completo de dramaturgos españoles contemporáneos, a cuya nómina ha hecho una aproximación Berta Muñoz, como se ha apuntado anteriormente. Dentro de la Biblioteca Virtual Miguel de Cervantes, encontramos portales más específicos, creados en convenios con Teatres de la Generalitat Valenciana y la Asociación de Teatro para la Infancia y la Juventud: la Biblioteca de Autores Valencianos o la Biblioteca ASSITEJ-España, respectivamente. A ellos nos referiremos, al cierre de este apartado, cuando se trate sobre otras posibles fuentes para la ampliación de este banco de autores y textos electrónicos.

\section{Centro de Documentación Teatral}

\section{(http://teatro.es/publicaciones)}

Dentro de la labor editorial del CDT, conjuntamente con la publicación en soporte papel han convivido también publicaciones digitales de textos dramáticos inéditos; alojados en su web, a fecha de julio de 2013, encontramos títulos ganadores del Premio Nacional de Teatro Calderón de la Barca de 2000, 2004, 2005, 2008 y 2009; de Pedro Víllora, Inmaculada Alvear, Antonio Rojano, Emiliano Pastor Steinmeyer y Blanca Doménech, respectivamente.

\section{Instituto de Tecnologías Educativas}

\section{(http://recursostic.educacion.es/apls/informacion_didactica/201/)}

La web Teatro de hoy del Instituto de Tecnologías Educativas (ITE), en su introducción al teatro español contemporáneo, entre otras finalidades, pretende acercar a los jóvenes los textos dramáticos actuales — de autores nacidos con posterioridad a 1950 (Muñoz Cáliz, 2011: 219)—. El objetivo es que las obras puedan servir de pauta a los montajes y ejercicios dramáticos de los talleres de teatro que se desarrollan en los centros escolares; y también de material de primera mano para los currículos de lengua y literatura de todos los niveles de secundaria. Aunque en menor grado que en castellano, no faltan obras en gallego y en catalán, con sus traducciones al castellano. La página ofrece también estudios particulares sobre la dramaturgia de algunos autores y un conjunto de actividades, entre las que destaca la lectura guiada de la obra. Aporta textos de más de medio centenar de autores: Albert Espinosa, Alfonso Plou, Alfonso Zurro, Antonio Álamo, Antonio Onetti, Beth Escudé i Gallés, Borja Ortiz de Gondra, Carles Batlle, Carmen Resino, Charo González Casas, David Desola, David Plana, David Planell, Ernesto Caballero, Eva Hibernia, Federico del Barrio, Fernando Renjifo, Ignacio Calvache, Ignacio del Moral, Ignacio García May, Inmacu- 
lada Alvear, Itziar Pascual, João Guisán, Jordi Galcerán, José Ignacio Juárez, José Ramón Fernández, Josep Pere Peyró, Juan Alberto López, Juan Carlos Rubio, Juan Copete, Juan Mayorga, Julio Salvatierra, Laila Ripoll, Lluïsa Cunillé, Luis García-Araus, Luis Merchán, Luis Miguel González, Manel Dueso, Marc Rosich, Margarita Sánchez, Maxi Rodríguez, Miguel Ángel Morillo, Miguel Murillo, Paco Paricio, Paco Zarzoso, Paloma Pedrero, Pau Miró, Sergi Belbel, Victoria Szpunberg, Xesús Pisón, Yolanda Dorado y Yolanda Pallín (a fecha de julio de 2013, cincuenta y dos autores; algunos de ellos hasta con más de un texto teatral digitalizado). Lozano Palacios (2013: 153-168) ha subrayado y comentado el interés infantil y juvenil de algunos de estos textos de literatura dramática.

\section{Muestra de Teatro Español de Autores Contemporáneos}

(http://www.muestrateatro.com/home.html\#pagina=/publicaciones.html)

Entre la información recogida en la página de la Muestra de Teatro Español de Autores Contemporáneos (MTAC), en el momento de redactar este artículo, se incluye la publicación de textos teatrales en dos colecciones virtuales, la denominada Dramaturgia Contemporánea, con textos de Juan Mayorga, Miguel Murillo, Itziar Pascual, Alejandro Jornet, Juan Luis Mira, Alfonso Plou, Maxi Rodríguez, Gracia Morales, Guillermo Heras, Pedro Montalbán, Antonio Cremades, Santiago Cortegoso, Jerónimo López Mozo, Francisco Sanguino, Lola Blasco y Borja Ortiz de Gondra; y la del Programa Dramaturgias Actuales, que incluye a Antonio Rojano, Alberto Conejero, José Manuel Mora, Jerónimo Cornelles, María Velasco y Jordi Faura. Entre los títulos, se recogen obras del teatro en otras lenguas en su traducción al castellano. Apunta Berta Muñoz (2012: 105-106) que los autores actuales españoles más relevantes han pasado por la Muestra y es uno de los repertorios biográficos más completos; con fecha de actualización de octubre de 2012, un total de cuatrocientos dieciocho autores y quinientas tres obras han sido representadas hasta su vigésima edición.

\section{Parnaseo}

\section{(http://parnaseo.uv.es/Ars/Autores/indexmonograficos.html)}

Web sobre Literatura Española de la Universidad de Valencia, estructurada en diferentes secciones, en una de ellas, Ars Theatrica Contemporánea, autodefinida como una ventana abierta a la nueva dramaturgia, acoge en sus monografías de autores contemporáneos la edición de los textos de los anexos de Stichomythia digitalizados. A fecha de julio de 2013, algo más de 
una decena de títulos, entre otros de Paco Zarzoso, Itziar Pascual, Raúl Hernández Garrido, Juan Mayorga, Ernesto Caballero y Angélica Liddell.

\section{Otras fuentes}

Además de las fuentes tenidas en cuenta en este artículo, otras permitirán ampliar la búsqueda de textos teatrales en formato digital. Desde páginas de ámbito más general a otras de ámbito geográfico más restringido, públicas o privadas, páginas de centros de documentación e investigación o de revistas, a modo de ejemplo, apuntamos:

En Dramática latinoamericana del Centro Latinoamericano de Investigación y Creación Teatral, CELCIT (http://www.celcit.org.ar/publicaciones/ dla.php), colección de textos relevantes de la dramaturgia iberoamericana contemporánea, con más de cuatrocientas obras procedentes de Argentina, Brasil, Chile, Colombia, Costa Rica, Ecuador, México, Perú, Puerto Rico, Uruguay, Venezuela..., encontramos textos de autores españoles: Juan Mayorga, Lluïsa Cunillé, Gracia Morales, Aurora Mateos, Raúl Hernández Garrido, Inmaculada Alvear, Paco Sanguino, Rafael González, Luis Miguel González Cruz, Itziar Pascual, Alfonso Sastre... Podrán leerse también obras de José Luis Alonso de Santos, Antonio Hernández, Carmen Pombero, Íñigo Ramírez de Haro, Manuel Veiga, José Sanchis Sinisterra, no incluidos en este trabajo por no encontrarse sus nombres entre las fuentes empleadas.

La Fundación Romea (http://www.fundacioromea.com/), a fecha de julio de 2013, mantiene en una de las secciones de su web un listado de doscientos treinta y siete dramaturgos contemporáneos, con ficha de más de quinientas obras en las que podemos consultar información de la lengua y ediciones; también, aunque no siempre, de la sinopsis o del número de personajes. El número de textos teatrales de este banco se reduce al examinarse la posibilidad de descarga o de lectura en Internet.

Aunque en buena parte los autores y obras de la Biblioteca de Autores Valencianos (http://www.cervantesvirtual.com/bib/portal/biblioteatral/) o de la Biblioteca ASSITEJ-España (http://www.cervantesvirtual.com/portales/ assitej/) aparecerán, al estar ya incluidos en la Biblioteca Virtual Miguel de Cervantes, sin embargo, los citamos aquí por su carácter más concreto y por el interés de posibles búsquedas de datos más específicos.

Adscrito a la Junta, el Centro de Documentación de las Artes Escénicas de Andalucía (http://www.juntadeandalucia.es/culturaydeporte/cdaea/), además de las publicaciones en papel, cuenta con distintas colecciones electrónicas. En la colección Teatro en Internet, ocho títulos, con textos en for- 
mato PDF, que incluyen, entre otros materiales, un estudio introductorio; también en otra colección digital, Teatro en Internet para Niños, pueden descargarse cuatro títulos; en Fuera de Colección, publicación con motivo del día mundial del teatro 2010, se recogen textos de pequeñas obras de teatro ${ }^{2}$ puestas en escena por alumnos y ex alumnos de la Escuela Superior de Arte Dramático de Sevilla, que transcurren todas ellas en la habitación de un hotel, piezas de una veintena de autores andaluces, coordinados por Alfonso Zurro, entre los no recogidos en el listado de autores de este trabajo, encontramos nuevos nombres para ampliar el inventario: Carlos Álvarez Ossorio, Carlos Álvarez-Nóvoa, Javier Berger, Antonio Estrada, Miguel Gallego, Javier García Teba, Antonio Hernández Centeno, Mercedes León, Fernando J. Mansilla, David Montero, José Moreno Arenas, Carmen Pombero, Antonio Raposo, Sergio Rubio y Adelardo Méndez Moya; junto a estos, los textos de otros autores andaluces que pueden consultarse en esta entrega, como los de Alfonso Zurro, Antonio Onetti, Antonio Rojano, Gracia Morales, Juan Carlos Rubio...

Aunque no permite la lectura en línea y la descarga directa, el portal Catalandrama (http://www.catalandrama.cat/), un proyecto del Institut Ramon Llull y la Sala Beckett para la difusión internacional del teatro catalán contemporáneo, recoge información sobre las traducciones de textos dramáticos catalanes de más de medio centenar de autores a otras lenguas, entre ellas el castellano, y ofrece la posibilidad de solicitar las traducciones vía Internet y de forma gratuita ${ }^{3}$.

En la revista La Ratonera, en formato papel y también en línea (http:// www.la-ratonera.net/), pueden leerse obras de una treintena de autores españoles, nombres conocidos dentro del panorama teatral asturiano junto con otros relevantes en el ámbito estatal e internacional, entremezclados en sus distintos números, desde el inicial de noviembre del 2000 hasta el número 37 de 2013: Laura Iglesia San Martín, Juan José Plans, José Luis Campal, Antonio Cremades, Pedro Montalbán, Josep Maria Miró i Coromina, Mayra Fernández, Alberto Conejero, Laila Ripoll, Carlos Be, Jorge Moreno, Maxi Rodríguez, Ernesto Caballero, Paco Zarzoso, Pilar Murillo, Adolfo Camilo Díaz, Roberto Corte, Eladio de Pablo, José Rico, Olga Cuervo, Carolina Barrios, Margarita García, Inma Rodríguez, Carmen Ruiz Tilve, Marta Fer-

2 Sobre el teatro breve tan presente en muchas de las piezas digitalizadas, cf. José Romera (ed., 2011). De la propuesta de Alfonso Zurro da cuenta pormenorizada María Jesús Orozco (2012: 347-359).

3 Un índice de autores y obras de la dramaturgia catalana contemporánea puede consultarse en la base de datos del Institut del Teatre (http://www.diba.es/sgae/). 
nández, Carmen Gómez Ojea, Boni Pérez, Raúl Hernández Garrido, Laureano Mántaras, Javier Villanueva y Fernando Arrabal.

La revista de investigación y creación teatral de la RESAD, Acotaciones (http://www.resad.com/acotaciones/archivo.htm), permite, en su sección Cartapacio — desde el número 21, de julio-diciembre de 2008-, leer o descargar textos completos de Lucía Vilanova, Lola Blasco, Marta Buchaca, Blanca Doménech, Diana I. Luque, Vanessa Montfort, Vanesa Sotelo, María Velasco, Alfredo Sanzol, Paco Zarzoso, Fernando Almena, Álvaro Custodio, José Martín Elizondo, José Ramón Fernández, Jesús Laiz, Yolanda Pallín, Laila Ripoll, Marco Antonio de la Parra, José Cruz, Jesús Campos García, José Luis Miranda, Ignacio Amestoy, Jerónimo López Mozo, así como numerosos fragmentos de otros autores en números anteriores a la fecha señalada.

El mismo SELITEN@T ha publicado una serie de textos teatrales: de Juan Mayorga, Cartas de amor a Stalin; de Pilar Campos, Selección natural y de Gracia Morales, Un horizonte amarillo en los ojos, todas ellas con edición en formato impreso (Madrid: Ediciones de la UNED) y digital en la revista Signa, en sus números 9 -para la obra del primero- y 16 -para los textos de las dos autoras-. Disponible en HTLM desde el portal de la revista (http://cervantesvirtual.com/hemeroteca/signa/catalogo.shtml).

La página de los Premios INJUVE (http://injuve.es/conocenos/ediciones-injuve/) acoge la edición electrónica de los textos ganadores y accésit del Premio Marqués de Bradomín, uno de los premios de escritura más importantes para los jóvenes dramaturgos. Creado en 1984 por Jesús Cracio, como director técnico del Instituto de la Juventud, y otorgado por primera vez en 1985 a Sergi Belver, está destinado a autores nacidos o residentes en España menores de treinta años cuyos textos hayan sido escritos en cualquiera de las lenguas del territorio español. En esta página, en el momento de redactar este trabajo, la publicación del catálogo del Premio Marqués de Bradomín alberga textos teatrales en castellano del 2008 al 2012, entre ellos, los de los ganadores: Emiliano Pablo Pastor Steinmeyer, Llàtzer García Alonso, Carlos Contreras Elvira, Enrique Olmos Avilés, Marcos Abalde Covelo, respectivamente.

Una última mención: las páginas de las propias compañías teatrales. Entre su diversa documentación —histórico, agenda, carteles, programas de mano, fotografías, vídeos, sinopsis, guías didácticas, dosieres con información artística y técnica, críticas sobre la recepción de las obras...- acogen, aunque en contadas ocasiones y de forma muy desigual, los textos de las 
obras; pero son tantas las que cuentan con página propia que ya de por sí requeriría ser objeto de otra investigación.

\subsection{Relación de abreviaturas, siglas y otros códigos empleados}

$\begin{array}{ll}\text { AAT } & \text { Asociación de Autores de Teatro. } \\ \text { aut* } & \text { Autoría compartida. } \\ \text { AVAE } & \text { Archivo Virtual de las Artes Escénicas. } \\ \text { CDT } & \text { Centro de Documentación Teatral. } \\ \text { CV } & \text { Cervantes Virtual. } \\ \text { ITE } & \text { Instituto de Tecnologías Educativas. } \\ \text { MTAC } & \text { Muestra de Teatro Español de Autores Contemporáneos. } \\ \text { o.p. } & \text { Otra página. } \\ \text { PA } & \text { Página de autor. } \\ \text { PA* } & \text { Página de autor sin textos en la fecha. } \\ \text { PARNASEO } & \text { Servidor web de la Universidad de Valencia. } \\ \text { PC } & \text { Página de compañía teatral. }\end{array}$

\section{DE LOS TEXTOS TEATRALES EN LAS PÁGINAS ELECTRÓNICAS DE LOS AUTORES}

ALMENA SANTIAGO, Fernando (Córdoba, 1943)

http://www.cervantesvirtual.com/bib/portal/aat/Almena/(AAT)

Boda del comecocos, La (AAT); Buenos malos tratos, Los (AAT); Cisne negro, El (AAT); Cuchipando, El (AAT); Cuidadores, Los (AAT); Ejercicios para ahuyentar fantasmas (AAT); ¡Gran guardabosque Gran! o la capicúa historia de un gerifalte gordo (AAT); Mandamás más más... y sus máquinas pitipitroncas, El (AAT); Misterio indescifrable, El (AAT); Piel del león, La (AAT); Redobles para un mono libre (AAT); Resurgimiento (AAT); Rito (AAT); Soledad del ejecutivo, La (AAT); Teatro para escolares (AAT); Tontos de capirote (AAT).

ASENSIO SOLAZ, Eugenio (Badalona, Barcelona, 1961)

https://sites.google.com/site/eugenioasensiosolaz/teatro (PA), (CV)

¿A dónde la llevo, señora?; Amnesia; Anciana sin hijos deja herencia por compartir últimos días (CV); Boleros en la cabeza (CV); Clase de lengua; Danza de la lluvia, La (CV); De Bagdad al cielo; Declaraciones en 
exclusiva; Deshielo (CV); Falta involuntaria; Homenaje; Institute for the Memory; Locus amoenus (CV); Nadie se acuerda ya de ti (CV); Paternidades (CV); Reencuentro; Reset (CV); Teatro versus teatro; Tengo Sida; Verdes praderas americanas, Las; Vulnerabilidad del viento, La; Y después....

BE, Carlos —vid. BLANCO GARCÍA, Juan Carlos

BLANCO GARCÍA, Juan Carlos (Barcelona, 1974) CARLOS BE

http://www.carlosbe.blogspot.com.es (PA)

Achicorias; Amén; Enemigos; Estamos abiertos a todas las familias; Extraordinaria muerte de Ulrike M., La; Jaque mate; Muere, Numancia, muere; Mujer más fea del mundo, La; My favorite things; Noel Road 25: a genius like us; Origami; Su vómito, el destino; Woman in a taxi crossing New York.

\section{BUENO MINGALLÓN, Antonia (Madrid, 1952)}

http://mujerdeteatro.blogspot.com.es (PA), (CV)

Arcángeles (CV); Aulidi (hijo mío); Malos días; Niña tumbada, La; Ópera de los residuos; Sancha: Reina de la Hispania; Todo por un duro; Tránsito; Tras los cristales; Zahra: favorita de Al-Andalus.

CAMPOS GARCÍA, Jesús (Jaén, 1938)

http://www.jesuscampos.com (PA)

http://www.cervantesvirtual.com/portales/jesus_campos/(AAT)

7.000 gallinas y un camello; A ciegas; A papel bien sabido, no hay cómico malo; Almas gemelas; Blancanieves y los siete enanitos gigantes; $\mathrm{Ca}$ beza del diablo, La; D. Juan@simetrico.es. (La burladora de Sevilla y el Tenorio del siglo XXI); Danza de la chatarra; Danza de la última pirámide; Danza de los veraneantes; Danza del primer recuerdo; Danza para violín y revólver; De compras; Depende; En un nicho amueblado; Entrando en calor; Entremeses variados; Es mentira; Es sólo una enfermedad; Famoseo, El; Fiera corrupia, La; Mando a distancia, El; Matrimonio de un autor teatral con la Junta de Censura; Me acuso de ser hetero; Nacimiento, pasión y muerte de... por ejemplo: tú; Naufragar en Internet; Noche de bodas; Número 17, La; Olor de las metáforas, El; Pareja con tenedor; Patético jinete del rock and roll; Pena y Pene; Posturas e imposturas; Ruleta rusa, La; Traje de cuero, El; Triple salto mortal con pirueta. 
CASANOVAS I GÜELL, Jordi (Vilafranca del Penedès, Barcelona, 1978) http://jordicasanovas.wordpress.com (PA)

Andorra; Revolución, La; Ruina, La.

CATALÁN GARCÍA, Pedro (Madrid, 1956)

http://www.cervantesvirtual.com/bib/portal/aat/Catalan/(AAT)

¡Y que cumplas muchos más!; Basura; Butterfly; Con la soga al cuello; Condenados, Los; Minimóvil; Poco de oxígeno por favor, Un; Trinchera, La.

CHAMIZO GÓMEZ, Patricio (Badajoz, 1936)

http://www.cervantesvirtual.com/bib/portal/aat/Chamizo/ (AAT)

Don Benito: (El crimen de Inés María); En un lugar de Alemania: drama de la emigración; Ganarás el pan con el sudor del de enfrente; Rudens: comedia musical.

COBALEDA COLLADO, Miguel (Salamanca, 1944)

http://www.cobaleda.net (PA)

http://www.cervantesvirtual.com/bib/portal/aat/Cobaleda/ (AAT)

Aliento y el barro, El; Forastero mineral profundo; Más allá del laberinto; Mensajero mudo, El; Papel impreso. Papel oprimido; Ramo para Isveth, Un; Serrallo, El.

ENRÍQUEZ MUÑOZ, Salvador (Granada, 1942)

http://salvadorenriquez.galeon.com (PA) ${ }^{4}$

http://www.cervantesvirtual.com/bib/portal/aat/Enriquez/(AAT)

¡No...: (didascalia de mimo para ser leída) (sic); ;Se busca!; 22 de diciembre; Agenda llena de grasa, Una; Ascensor, El; Bajo un pubis primerizo; Cara oculta de la humanidad, La; Compás de dos por cuatro; Cuando den las tres; Cuchara, La; Diálogo de los ausentes; En la distancia; Hombre que no vio la mar, El; Le llamaban Kafka; Mirándose detrás de un espejo;

${ }^{4}$ La página web Noticias teatrales (http://noticiasteatrales.es) que edita este autor e investigador teatral, en su recopilación, no solo de sus obras, sino también de las de otros autores, abre las puertas a un incremento muy significativo en ampliaciones futuras del catálogo de nombres y títulos aquí propuestos. 
No hay precio... el que tú quieras; Periódico en blanco, Un; Próxima, prosperidad, La; Puente, El; Reality show; Sólo para mayores; Yo, pecador.

ESCABIAS, Juana — vid. ESCABIAS TORO, Juana Natividad

ESCABIAS TORO, Juana Natividad (Madrid, 1964) JUANA ESCABIAS http://www.juanaescabias.es (PA)

Fantasmas; Historia de un imbécil; Hojas de algún calendario; Ícaros; Interiores; Invisibles; Islas; Parejas.

ESCALADA MUÑOZ, Julio (Madrid, 1963)

http://www.julioescalada.com/ (PA)

Cuento de Navidad; En el borde; Invierno (Cuatro estaciones); Otoño (Cuatro estaciones); Primavera (Cuatro estaciones); Principito, El; Sois la bomba; Te vas, me dejas y me abandonas; Verano (Cuatro estaciones).

ETXEBA, Carlos —vid. SÁEZ ECHEVARRÍA, Carlos

GALÁN, Marta (Barcelona, 1973)

http://www.marta-galan.com (PA)

Lola (AVAE); Machos (AVAE); Melodrama (AVAE); Perro, El (AVAE); Protégeme, instrúyeme; Transilvania 187, in memoriam (AVAE).

GARCÍA RUIZ, Carlos (Ponferrada, 1971) CARLOS RUIZ

http://www.carlosgr.net (PA)

Tigre de Usera, El (CV).

GARCÍA VALLEJO, Alfonso (Santander, 1943) ALFONSO VALLEJO http://www.alfonsovallejo.com (PA)

http://www.cervantesvirtual.com/bib/bib_autor/alfonsovallejo/(AAT)

A tumba abierta; Ácido sulfúrico; Cangrejos de pared; Cero transparente, El; Crujidos; Culpable ¿Pssss...; Desguace; Ebola Nerón; Eclipse; Escuchador de hielo, El; Espacio interior; Espalda del círculo, La; Fly-By; Gaviotas subterráneas; Hiroshima-Sevilla. 6A; Hölderlin; Infratonos; Jindama; Ka-OS; Katacumbia; Kora; Latidos; Monkeys; Monólogo para seis voces sin sonido; Nueva mujer, Una; Orquídeas y Panteras; Panic; Sol ulcerado; Tuatú; Week-end. 
Autores y TEXTOS TEATRALES ELECTRÓNiCOS (EN CASTELlANO)...

GODOY MARQUET, Antonio (Barcelona, 1966) TONI MARQUET http://www.cervantesvirtual.com/bib/portal/aat/Tonimarquet/(AAT) Ausencias.

GONZÁLEZ GOSÁLBEZ, Rafael (Alicante, 1966)

http://www.cervantesvirtual.com/bib/portal/aat/Gonzalez/obras.shtml (AAT)

Penalty de Panenka, El; Pesadilla, La; Creo en Dios (aut* —vid. SANGUINO, Francisco) (CV); Metro (aut* — vid. SANGUINO, Francisco) (CV).

HERNÁNDEZ GARRIDO, Raúl (Madrid, 1964)

http://hernandezgarrido.com/(PA)

http://www.cervantesvirtual.com/bib/portal/aat/hernandez/(AAT)

Calibán; Eclipses; Esclavos: Los engranajes, Los (PARNASEO); Esclavos: Los malditos, Los; Esclavos: Los restos: Agamenón vuelve a casa, Los; Esclavos: Los restos: Fedra, Los; Fotos; Juego de dos: la persistencia de la imagen; Madres de mayo van de excursión, Las; Partículas elementales; Si un día me olvidaras; Soto voce; Sueños de la ciudad, Los; Tábano y la ara$\tilde{n} a ;$ Te mandaré una carta.

IGLESIAS SIMÓN, Pablo (Madrid, 1977)

http://www.pabloiglesiassimon.com (PA)

11-N (CV); Alicia frente al espejo (CV); Lado oeste del Golden Gate, El (CV); Sin móvil aparente (CV); Tu imagen sola (aut* — vid. ORTIZ DE GONDRA, Borja) (CV).

LALANA, Fernando (Zaragoza, 1958)

http://www.fernandolalana.com (PA)

Viaducto de los suicidas, El.

LÓPEZ MOZO, Jerónimo (Girona, 1942)

http://www.cervantesvirtual.com/bib/bib_autor/jeronimolopez/(AAT)

Ahlàn; Arquitecto y el relojero, El; Bajo los rascacielos (Manhattan cota_20); Combate de ciegos; Ella se va; Eloídes: obra dramática dividida en XXVIII escenas; En aquel lugar de la Mancha; Engaño a los ojos, El; 
Guernica; Hijos de Hybris; Macbeth, Los; Matadero solemne; Olvido está lleno de memoria, El; Raíces cortadas, Las.

MAESTRO LUENGO, José Alberto (Madrid, 1964)

http://www.cervantesvirtual.com/bib/portal/aat/Josemaestro/index.shtml (AAT)

Realidad es otra, La.

MANAUT, Stella (Madrid, 1941)

http://www.stellamanaut.es (PA)

Amor sin propiedad privada; Cada mochuelo, a su olivo; Casa de la Bernarda, La; Cenizas del difunto, Las; Cita a ciegas; Coro de asesinas; Doña Obdulia, el marido y su hermana doña olvido; Dos ancianos de buen ver; Francamente, la música me conmueve; Mi padre es un hombre muy, pero que muy importante; Mujer de silicona, La; Niño probeta, El; Pagando, oigan; pagando; Revolución de las pepas, La; San Antonio de la Flori$d a$; Teatro en porciones (CV); Tiempo de los sauces (CV), El; Titi aborrecible, la abuela ninfómana y el lobo gay; Torturada; Velada romántica, Una; Velando a un muerto que tenía una mosca en la punta de la nariz.

MARQUET, Toni — vid. GODOY MARQUET, Antonio

MARTÍN BERMÚDEZ, Santiago (Madrid, 1947)

http://www.cervantesvirtual.com/bib/bib_autor/bermudez/(AAT)

Carmencita revisited; Dalila y los virtuosos; Garcilaso, coloquio y silencio; Gradas de San Felipe, Las; Lunas; Más fingida ocasión y Quijotes encontrados, La; No faltéis esta noche; Nosotros, que nos quisimos tanto; Pasión-papel; Penas de amor prohibido; Solimán y la reina de los pequeños; Tiresias, aunque ciego; Vals de los condenados, El.

MARTÍN SALINAS, Isabel (Almería, 1957)

http://isabelmartinsalinas.blogspot.com.es/(PA)

Collar de cerezas (CV); Hispania, Hispania; Hoyo 18, El; Río abajo; Rita; Segundas partes; Soplo de viento, Un; Verano del membrillo.

MATEOS RODRÍGUEZ, Aurora (Colmenar, Málaga, 1974)

http://auroramateos.com (PA) 
Agua; Algunas notas sobre la anormalidad; Amigo de medianoche, El; Articulo 153; Chinaboy (El niño chino); Et in hora (CV); Eternidad y otras mentiras, La; Memorias de un mundo perdido; Rafael y la Fornarina; Suicidio del ángel, El.

MILLÁS MASCARÓS, Ana Amparo (Valencia, 1958)

http://anamillasteatro.galeon.com (PA)

¡Simpelen, a mi pesar...! (CV); ¿Soy culpable? (CV); Agua de celos (CV); Al pie de la letra (CV); Ángela caída (CV); Duda, La (CV); Europa o la humanidad nunca aprenderá (CV); Hombre que contaba (CV), El; Nómada en el tiempo (CV); Terrae vitis (CV).

MIR MALUQUER, Francisco de Paula (Barcelona, 1957) PACO MIR

http://www.cervantesvirtual.com/bib/portal/aat/Mir/(AAT)

Carta dudosa; Don o maldición; Informativos; Neonatos; No es tan fácil; Regidor

MIR, Paco — vid. MIR MALUQUER, Francisco de Paula

MIRA, Juanluis — vid. MIRA CANDEL, Juan Luis MIRA

MIRA CANDEL, Juan Luis (Orihuela, Alicante, 1955) JUAN LUIS

http://juanluismira.com (PA)

http://www.cervantesvirtual.com/bib/portal/aat/Mira/ (AAT)

A ras del cielo; Aduosadas; Alici@.com; Blues de Macky Navaja, El; Cor de mel; Cosas...; De Lope; Esperando el bus; Felices años veinte, Los; Femenino plural; Litrona; Malsueño; Mañana será otro milenio; Maquillando cadáveres; Mar de almendros; Mar en el bolsillo: (la familia Glub), El; Mi amigo Bart; Noche de perros y girasoles; Noche menos pensada, La; Ojos de lluvia; Rey Volpone; Vida silenciosa de los Ácaros, La.

MIRALLES GRANCHA, Alberto (Alicante, 1940 - Madrid, 2004)

http://www.cervantesvirtual.com/portales/alberto_miralles/(AAT)

¡Hay motín, compañeras!; Amantes del demonio, Los; Aventuras, misterios y maravillas del rey Arturo y los caballeros de la mesa redonda; Céfiro agreste de olímpicos embates; Centellas en el sótano del museo; Colón. Versos de arte menor por un varón ilustre; Felicidad de la piedra, La; Fies- 
ta de los locos, La; Guerra. El hombre, La; Héroes mitológicos; Jardín de nuestra infancia, El; Juegos prohibidos (El crepúsculo del paganismo romano); Metempsicosis; Okupas en el Museo del Prado; Píntame en la eternidad; Teatro breve; Trino del diablo, El; Último dragón del Mediterráneo, El.

OMAR WALLS, Alberto (Tenerife, 1943)

http://www.albertoomarwalls.com (PA)

¡Dos coleópteros y un amor!

ONETTI, Antonio (Sevilla, 1962)

http://antonioonetti.com (PA)

Amar en tiempos revueltos; Chica de cristal, La; Diva al dente, La; Líbrame, señor, de mis cadenas (CV); Madre caballo; Malfario (CV); Marcado por el típex; Nadia, o los enanos van creciendo; Naturaleza muerta; Peligros de la jungla, Los; Puñalá, La; Rave party; Romeo x Julieta; Salvia; Solas; Son que nos tocan, El.

ORTIZ DE GONDRA, Borja ((Bilbao, 1965)

Borja; memento mori (MTAC); Herida en la voz (ITE); Tu imagen sola (aut* — vid. IGLESIAS SIMÓN, Pablo) (CV).

PASCUAL ORTIZ, Itziar (Madrid, 1967)

http://www.cervantesvirtual.com/bib/portal/aat/Itziar/(AAT)

Domador de sombras, El (ITE); Mascando ortigas; Père Lachaise; Solo tres palabras (PARNASEO); Variaciones sobre Rosa Park (MTAC); Voces de Penélope, Las (PARNASEO).

PLOU, Alfonso (Zaragoza, 1964)

http://www.cervantesvirtual.com/bib/bib_autor/alfonsoplou/(AAT)

Buñuel, Lorca y Dalí; Lucha a muerte del zorro y el tigre (MTAC); Macbeth \& Lady Macbeth; Picasso adora la Maar (sic); Volcán y la marea, El (ITE).

POLO BARRENA, Juan (Madrid, 1936)

http://www.cervantesvirtual.com/bib/portal/aat/polo/ (AAT)

Delicada y exquisita desaparición de un hombre, La; Invitado de honor; María Dubrovska, primera actriz; Muerte de Brunelda, La. 
ROJANO MORA, Antonio José (Córdoba, 1982)

http://antoniorojano.wordpress.com (PA)

$B$ is for bacon (CV); Decadencia en Varsovia, La; Despedida sin título; Jacqueline K.; Katiuskas (MTAC); Línea en la arena, La; Magnífica desolación, Una; Ocho segundos en la mente de un cowboy adolescente; Sueños de arena; Yo también camino como Jayne Mansfield.

ROMERO FERNÁNDEZ, Francisco (Ciudad Real, 1961)

http://www.ebaobab.com/(PA)

http://www.cervantesvirtual.com/bib/portal/aat/Romero/index.shtml (AAT)

Censura; Hasta que la vida nos separe; Interrogatorio, El; Jubil acción; Legado de Julie Newman, El; Liquidación total; Manual de erudición para famosos; Posadera, La; Puto teatro; Quimera de Alonso y Sancho, La; Se precisa hombre bien dotado para importante labor social; Shylock Gallerie; Terapia; Último amanecer, El; Último paraíso de Cándido, El.

RUBIO CRUZ, Juan Carlos (Córdoba, 1967)

http://www.juancarlosrubio.com (PA)

10 (CV); ¿Dónde se esconden los sueños? (CV) (ITE); $100 \mathrm{~m}^{2} / \mathrm{el}$ inconveniente; 9 minutos/epitafio; Adrenalina, feniletilamina, oxitocina y otras hormonas (CV); Arizona; Epitafio (CV); Esta noche no estoy para nadie (CV); Heridas del viento, Las (CV) (ITE); Humo (CV); Tres (CV).

RUIZ NEGRE, Antonio (Valencia, 1938)

http://www.ruiznegre.com (PA)

¡Jo, qué familia! (CV); ;Vaya una historia! (CV); A este lado de la línea (CV); A ningún sitio (CV); Alzar el vuelo (CV); Capricho, Un (CV); Como un dulce muy amargo (CV); De corazón (CV); Denario de cobre (CV); Divorciadas $(\mathrm{CV})$; El hostal del laurel, El (CV); Él no lo haría $(\mathrm{CV})$; Fondos reservados (CV); Maldito seas (CV); Mamá, quiero ser furcia (CV); Martes otra vez, El $(\mathrm{CV})$; Me vuelvo a casa $(\mathrm{CV})$; Nunca más en jueves $(\mathrm{CV})$; Paella de mariscos (CV); Secuestradas (CV); Trío de hecho (CV); Yo, en mi casa (CV).

RUIZ, Carlos — vid. GARCÍA RUIZ, Carlos

SÁEZ ECHEVARRÍA, Carlos (Bilbao, 1929) CARLOS ETXEBA

http://www.cervantesvirtual.com/bib/portal/aat/Etxeba/ (AAT) 
Adivino oriental, El; Alcaldesa feminista, Una; Aldeana aprovechada, Una; Amor a los hijos, El; Aspasia o la liberación de la mujer; Bofetón y el ombligo: (monólogo teatral), El; Canario de Macario, El; Cartas anónimas; Cerca del Gólgota; Cómo cazar maridos; Cómo engañar a las mujeres; Conde Drácula tiene SIDA, El; Consultorio sexológico femenino; Crisis del teatro, La; Cuerda del reloj, La; D. Ángel Bueno: (monólogo teatral con cuatro actores mudos); Diablo cojuelo de los ladrones: (espectáculo teatral), El; Diván, El; Estrella de cine, Una; Fea, La; Feligresa impenitente, Una; Flechazo, El; Herencia del abuelo, La; Historia mínima de un recién nacido en Mozambique en el año 2000; Lo que no son pesetas son puñetas; Lope de Lepe, El; Lord inglés, Un; Mala vida: (monólogo), La; Mantis irreligiosa, La; Merengue con coco; Monólogo del mendigo; Monólogo total; Mujeres de Mahoma, Las; Música de piedra; Pitonisa oriental y Bin Laden, La; Portería: (monólogo teatral), La; Rascacielos, El; Sanguinaria, La; Señora de la limpieza, Una; Tía Tula se hace feminista, La; Trabajo fijo (teatro breve), El; Yo fui novio de la Lewinsky; Yo, Judas Iscariote; Zapapote-cenozoicosaurio (minitragedia universal zapapotética en dos partes), El.

SANGUINO, Francisco (Alicante, 1964) PACO SANGUINO

Creo en Dios (aut* —vid. GONZÁLEZ GOSÁLBEZ, Rafael) (CV); Incertidumbre (MTAC); Metro (aut* —vid. GONZÁLEZ GOSÁLBEZ, Rafael) (CV).

SANGUINO, Paco — vid. SANGUINO, Francisco

SASTRE SALVADOR, Alfonso (Madrid, 1926)

http://www.sastre-forest.com (PA*)

http://www.cervantesvirtual.com/bib/bib_autor/alfonsosastre/(AAT)

Fama y olvido.

VALLEJO, Alfonso — vid. GARCÍA VALLEJO, Alfonso

VÍLLORA GALLARDO, Pedro Manuel (Albacete, 1968) PEDRO VÍLLORA

http://www.pedrovillora.com/(PA)

Bésame macho (CDT); Mayor; Muerte, La.

VÍLLORA, Pedro — vid. VÍLLORA GALLARDO, Pedro Manuel 


\section{TEXTOS TEATRALES EN OTRAS PÁGINAS ELECTRÓNICAS}

ÁLAMO, Antonio (Córdoba, 1964) (ITE)

ÁLVAREZ FERNÁNDEZ, Miguel Ángel (1963) (CV)

ALVEAR, Inmaculada (ITE) (PC)

BARRIO, Federico del (Madrid, 1957) (ITE)

BATLLE JORDÁ, Carles (Barcelona, 1963) (ITE)

BELBEL COSLADO, Sergi (Terrassa, Barcelona, 1963) (ITE)

BELMONTE AGÜERA, Rafael (Cartagena, 1956?) (CV)

BERNAT, Roger (Barcelona, 1968) (AVAE)

http://rogerbernat.info (PA*)

BLASCO MENA, Lola (Alicante, 1983) (MTAC)

http://lola-blasco.blogspot.com.es (PA*)

BOADELLA ONCINS, Albert (Barcelona, 1943) (CV)

BUDIA, Mariam (Logroño, 1970) (CV)

CABALLERO, Ernesto (Madrid, España, 1958) (ITE) (PARNASEO)

CALINCA, Tadeus (Xàtiva, Valencia, 1968) (PARNASEO)

http://tadeuscalinca.blogspot.com.es (PA*)

CALLE SORIANO, Poli (Puertollano, Ciudad Real, 1965) (CV)

CONEJERO LÓPEZ, Alberto (Jaén, 1978) (MTAC)

COPETE FERNÁNDEZ, Juan (Mérida, 1961) (ITE)

CORNELLES, Jerónimo (Argentina, 1976) (MTAC)

http://www.jeronimocornelles.es (PA*)

CORTEGOSO CALVAR, Santiago (Moaña, Pontevedra, 1974) (MTAC)

CREMADES CASCALES, Antonio (Alicante, 1960) (MTAC)

CUNILLÉ SALGADO, Lluïsa (Badalona, Barcelona, 1961) (ITE)

DESOLA MEDIAVILLA, David (Barcelona, 1971) (ITE) 
DOMÉNECH CASARES, Blanca (Madrid, 1976) (CDT)

DORADO AGUILAR, Yolanda (Córdoba, 1969) (ITE)

DUESO, Manel (Barcelona, 1953) (ITE)

ESCUDÉ I GALLÉS, Beth —vid. ESCUDÉ I GALLÉS, Elisabeth

ESCUDÉ I GALLÉS, Elisabeth (Barcelona, 1963) (ITE)

ESPINOSA I PUIG, Albert (Barcelona, 1973) (ITE)

FAURA, Jordi (Sabadell, Barcelona, 1982) (MTAC)

FERNÁNDEZ, José Ramón (Madrid, 1962) (ITE) (PARNASEO) (PC)

GALCERÁN I FERRER, Jordi (Barcelona, 1964) (ITE)

GARCÍA LARRONDO, Juan (Puerto de Santa María, Cádiz, 1965) (CV)

GARCÍA MAY, Ignacio (Madrid, 1965) (ITE)

GARCÍA ZURRO, Alfonso (Salamanca, 1953) (ITE) ALFONSO ZURRO

GARCÍA-ARAUS, Luis (Madrid, 1970) (ITE)

GONZÁLEZ CASAS, Charo (Madrid, 1964) (ITE)

GONZÁLEZ CRUZ, Luis Miguel (Cáceres, 1965) (CV) (ITE) (PARNASEO) (PC)

GONZÁLEZ, Angélica (Figueres, Girona, 1966) (AVAE) (PARNASEO) ANGÉLICA LIDDELL

http://www.angelicaliddell.com (PA*)

GUERRA FERNÁNDEZ DE ARANGUIZ, Alicia (1938) (CV)

GUISAN SEIXAS, João (A Coruña, 1957) (ITE)

HERAS GONZÁLEZ, Juan Pablo (Madrid, 1979) (CV) (o.p.) ${ }^{5}$

HERAS TOLEDO, Guillermo (Madrid, 1952) (CV) (MTAC)

HIBERNIA, Eva (Logroño, 1973) (ITE)

JIMÉNEZ PALO, Javier (Madrid, 1975) (CV)

5 En el listado del Portal de Dramaturgos (http://www.portaldedramaturgos. com.ar/jpheras/), espacio dedicado a los dramaturgos argentinos con el apoyo de la Secretaría de Cultura de Buenos Aires. 
JORNET, Alejandro (Valencia, 1956) (PARNASEO)

JUÁREZ, José Ignacio (Zaragoza, 1958) (ITE)

LENZA GONZÁLEZ, Enrique (Madrid, 1928) (CV)

LIDDELL, Angélica — vid. GONZÁLEZ, Angélica

LÓPEZ RODRÍGUEZ, Juan Alberto (Sevilla, 1962) (ITE)

MAQUA LARA, Javier (Madrid, 1945) (CV)

MARQUERIE, Carlos (Madrid, 1954) (AVAE)

MARSILLACH SORIANO, Adolfo (Barcelona, 1928 - Madrid, 2002) (CV)

MARTÍNEZ PARAMIO, Agapito (Zamora, 1962) (CV)

MAYORGA RUANO, Juan (Madrid, 1965) (ITE) (MTAC) (PC)

MERCHÁN DONAIRE, Luis (Caspe, Zaragoza, 1962) (ITE)

http://luismerchan.blogia.com (PA*)

MIRANDA ROLDÁN, José Luis (Málaga, 1939) (CV)

MIRAS MOLINA, Domingo (Ciudad Real, 1934) (CV)

MIRÓ CAPARROS, Pau (Barcelona, 1974) (ITE)

MONTALBÁN KROEBEL, Pedro (São Paulo, Brasil, 1961) (MTAC)

http://www.montalban-kroebel.com (PA*)

MORA ORTIZ, José Manuel (Sevilla, 1978) (MTAC)

MORAL ITUARTE, Ignacio del (San Sebastián,1956) (CV) (ITE)

MORALES ORTIZ, Gracia (Motril, Granada, 1973) (CV) (MTAC) (PC)

MORILLO, Miguel Ángel (Madrid, 1975) (ITE)

MURILLO, Miguel (Badajoz, 1953) (ITE) (MTAC)

NAVARRO I SALVADOR, Josep (Valencia, 1945) (CV)

PALLÍN, Yolanda (Madrid, 1965) (ITE)

PARICIO, Paco (Binéfar, Huesca) (ITE)

PEDRERO DÍAZ-CANEJA, Paloma (Madrid, 1957) (ITE)

PEYRÓ, Josep Pere (Palma de Mallorca, 1959) (ITE) 
PINTO CAÑÓN, Ramiro (Madrid, 1961) (CV)

PISÓN VILLAPOL, Xesús (Lugo, 1954) (ITE)

PLANA, David (Barcelona, 1969) (ITE)

PLANELL, David (Madrid, 1967) (ITE)

PUCHADES, Xavier (Valencia, 1973) (PARNASEO)

RENJIFO, Fernando (Madrid, 1972) (AVAE) (ITE)

RESINO DE RON, Carmen (Madrid, 1941) (CV) (ITE)

http://www.carmenresino.com/ (PA*)

RIPOLL CUETOS, Laila (Madrid, 1964) (ITE)

RODRÍGUEZ MÉNDEZ, José María (Madrid, 1925-2009) (CV)

RODRÍGUEZ, Maxi (Asturias, 1965) (ITE) (MTAC)

http://www.maxirodriguez.es (PA*)

ROSICH, Marc (Barcelona, 1973) (ITE)

SALVATIERRA CUENCA, Julio (Granada, 1964) (ITE)

SÁNCHEZ VELASCO, Arturo (Castellón, 1974) (PARNASEO)

SÁNCHEZ, Margarita (Madrid, 1962) (ITE)

SARRIAS, Mercè (Barcelona, 1966) (PARNASEO)

SIGNES MENGUAL, Miguel (Alicante, Valencia, 1935) (CV)

SZPUNBERG, Victoria (Buenos Aires, 1973) (ITE)

TOQUERO SANDOVAL, Carlos (Valladolid, 1946) (CV)

VALDERRAMA MODRÓN, Luis (Palencia, 1933) (CV)

http://www.valderrama-modron.com (PA*)

VÁZQUEZ, Gerard (Barcelona, 1959) (PARNASEO)

VELASCO, María (Burgos, 1984) (MTAC)

ZARZOSO, Paco (Valencia, 1966) (ITE) (PARNASEO)

ZURRO, Alfonso — vid. GARCÍA ZURRO, Alfonso 


\section{A MODO DE CIERRE}

Se recogen un total de cuatrocientos ochenta y ocho textos teatrales de cuarenta y cuatro autores en el epígrafe dos. Esta nómina de dramaturgos con textos en la Red se amplía hasta un total de ciento treinta y cuatro, si además de las propias páginas del autor se consideran otras fuentes, las indicadas en el apartado correspondiente. Esta ampliación del listado de autores se recoge en el epígrafe tres. Por los datos obtenidos, el principal portal para acceder de forma gratuita a obras teatrales a texto completo es la Biblioteca Virtual Miguel de Cervantes.

De los cuarenta y cuatro nombres cuyos títulos de los textos aparecen recogidos, veinte de ellos - al margen de aquellos con portal en la Biblioteca Virtual Miguel de Cervantes, a los que nos referiremos luego-cuentan con página propia. Se suman a los cuarenta y cuatro dos nombres que carecen de página, pero que se incluyen por autoría compartida con quienes cuentan con ella, estas dos excepciones son Borja Ortiz y Francisco Sanguino. Estos veinte autores con página personal son: Eugenio Asensio, Carlos Be, Antonia Bueno, Jordi Casanovas, Juana Escabias, Julio Escalada, Marta Galán, Carlos Ruiz, Pablo Iglesias Simón, Fernando Lalana, Stella Manaut, Isabel Martín, Aurora Mateos, Ana Millás, Alberto Omar, Antonio Onetti, Antonio Rojano, Juan Carlos Rubio, Antonio Ruiz y Pedro Víllora. En buena parte, los textos recogidos de estos autores proceden de sus páginas personales y se complementan con otras externas. En ocasiones, las obras aparecen enlazadas para su lectura o descarga desde la página personal con la de la autora o autor en la Biblioteca Virtual Miguel de Cervantes, así, por ejemplo, en casos como los de Antonia Bueno o Pablo Iglesias Simón.

En el apartado con página electrónica propia, se han incluido aquellos nombres con página de autor del catálogo de AAT. A fecha de julio de 2013, de los veintitrés nombres con biblioteca de autor, veintidós de ellos ponen sus obras para su difusión y a disposición del público: Alberto Miralles, Alfonso Plou, Alfonso Sastre, Alfonso Vallejo, Carlos Etxeba, Fernando Almena, Francisco Romero, Itziar Pascual, Jerónimo López Mozo, Jesús Campos García, José Maestro, Juan Luis Mira, Juan Polo Barrena, Miguel Cobaleda Collado, Paco Mir, Patricio Chamizo, Pedro Catalán, Rafael González, Raúl Hernández Garrido, Salvador Enríquez, Santiago Martín Bermúdez y Toni Marquet. De estos autores con portal en la Biblioteca Virtual Miguel de Cervantes, siete de ellos cuentan además con otra página personal, caso de Alfonso Sastre, Alfonso Vallejo, Francisco Romero, Jesús Campos, Juan Luis Mira, Miguel Cobaleda, Raúl Hernández y Salvador Enrí- 
quez. En el tema que nos ocupa, la posibilidad de lectura y descarga de los textos de estas páginas personales es desigual, y se mueve entre la no o casi nula posibilidad y el poder beneficiarnos de la generosidad de autores como Alfonso Vallejo, Jesús Campos, Salvador Enríquez o Raúl Hernández que ponen en su totalidad o en gran parte su obra a disposición de los interesados. De los veintitrés nombres del citado catálogo, referido al principio de párrafo, se exceptúa el portal y biblioteca de Antonio Buero Vallejo (1916-2000) con abundantes materiales e información sobre sus obras teatrales, pero no cuenta con muestras de sus textos teatrales completos.

Por razones de propiedad, enlaces rotos..., cabe señalar aquellas páginas de autores relevantes que carecen de obras completas, en el mejor de los casos contamos con extractos o fragmentos; significativas ausencias en: J. L. Alonso de Santos (http://www.joseluisalonsodesantos.com/), F. Arrabal (http://www.arrabal.org), L. Blasco (http://lola-blasco.blogspot.com.es), A. Gala (http://www.antoniogala.es/), R. García (http://rodrigogarcia.es), A. Liddell (http://www.angelicaliddell.com), F. Nieva (http://www.francisconieva.com/), C. Resino (http://www.carmenresino.com/) o Í. Ramírez de Haro (http://www.ramirezdeharo.com/), por ejemplo. En algunos casos es muy difícil encontrar textos publicados en la Red, ni en sus páginas personales, cuando cuentan con ella, ni en páginas ajenas. Ejemplo de ello: José Luis Alonso de Santos, Fernando Arrabal, Antonio Gala, Francisco Nieva, Sanchis Sinisterra, etc.

Si vivir de la literatura es difícil y aún más de la escritura teatral, frente a lo expuesto en el párrafo precedente, es encomiable la recogida de textos en páginas personales que permiten la lectura y descarga, al tiempo que se visibiliza su obra, tanto en las de autores veteranos como en las de los más jóvenes, como en las de Jesús Campos, Antonio Rojano, Alfonso Vallejo, o en la web, citada a pie de página, de Salvador Enríquez, ya no solo en lo que a su obra se refiere sino también por la aportación del excelente banco de textos de otros autores.

En la totalidad de los casos vistos, vivos o fallecidos en este siglo XXI, encontramos nombres de la literatura dramática de diferentes edades y promociones; así, nacidos antes de los años cincuenta del siglo anterior, veintisiete; en la década de los cincuenta, veintitrés; en la de los sesenta, cincuenta y uno; en la de los setenta, veintiséis; y solo cinco nuevos valores, en la de los ochenta. Generacionalmente, los autores nacidos en los años cincuenta o anteriores suponen el 38\%, igual porcentaje los de los sesenta y un 24\% los nacidos en los setenta y ochenta. Estos porcentajes que tienden a ser 
iguales en los grupos de edad señalados, aunque algo menor en los más jóvenes, no permiten concluir con grandes diferencias por este criterio, pero todo parece indicar que, con excepciones, en el uso de las nuevas tecnologías al servicio de acceso a sus obras: «son precisamente aquellos [autores] que más publican y los más innovadores los que también cuentan con estas herramientas en Internet [...] son aquellos autores más consolidados o que observan la dramaturgia como una forma de sustento vital de primer orden», como ha señalado Fernando Olaya (2013: 143), o muy en relación con el grado de profesionalización, al que yo mismo me he referido en el análisis de la presencia en Internet de las compañías gallegas (2013: 316).

Espero que la intención inicial, de aproximación a los autores del teatro español contemporáneo y a sus obras a texto completo en versión electrónica disponibles para la lectura y descarga gratuita, así como a las fuentes citadas y a algunas otras posibles que pudiesen facilitar nuevas aportaciones, se hayan cubierto y favorezcan ese incremento en futuras investigaciones. Por ello, lejos de cerrarse, este corpus de materiales de nombres y textos teatrales es un punto y seguido. En cuanto se indaga en otras fuentes, nuevos nombres aparecen con sus textos: Marcos Abalde Covelo, José Luis Alonso de Santos, Carlos Álvarez Ossorio, Carlos Álvarez-Nóvoa, Ignacio Amestoy, Fernando Arrabal, Javier Berger, Marta Buchaca, Pilar Campos, Carlos Contreras Elvira, José Cruz, Marco Antonio de la Parra, Antonio Estrada, Pedro Fresneda, Miguel Gallego, Javier García Teba, Llàtzer García, Antonio Hernández Centeno, Mercedes León, Fernando J. Mansilla, Adelardo Méndez Moya, David Montero, José Moreno Arenas, Enrique Olmos Avilés, Carmen Pombero, Íñigo Ramírez de Haro, Antonio Raposo, Sergio Rubio, José Sanchis Sinisterra, Alfredo Sanzol, Vanesa Sotelo, Manuel Veiga...

\section{REFERENCIAS BIBLIOGRÁFICAS}

ABUÍN GONZÁLEZ, A. (2008). «Teatro y nuevas tecnologías: conceptos básicos». Signa. Revista de la Asociación de Semiótica 17, 29-56 (disponible también en http://www.cervantesvirtual.com/obra/teatro-y-nuevastecnologas-conceptos- bsicos-0/).

DE LA TORRE RODRÍGUEZ, R. (2013). «La presencia en Internet de compañías de teatro gallegas (2000-2009)». En Teatro e Internet en la primera década del siglo XXI, José Romera Castillo (ed.), 307-319. Madrid: Verbum. 
LOZANO PALACIOS, M. I. (2013. «Literatura dramática infantil y juvenil actual e Internet». En Teatro e Internet en la primera década del siglo XXI, José Romera Castillo (ed.), 153-168. Madrid: Verbum.

MUÑOZ CÁLIZ, B. (2011). Fuentes y recursos para el estudio del teatro español. I. Mapa de la documentación teatral en España. Madrid: Centro de Documentación Teatral.

- (2012). Fuentes y recursos para el estudio del teatro español. II. Guía de obras de referencia y consulta. Madrid: Centro de Documentación Teatral.

- (2013). «Internet e investigación teatral. Fuentes en la red para la investigación del teatro español de los siglos XIX y XX». En Teatro e Internet en la primera década del siglo XXI, José Romera Castillo (ed.), 3360. Madrid: Verbum.

OLAYA PÉREZ, F. (2013). «La dramaturgia española actual en castellano: algunas calas sobre la edición de sus textos en España y su presencia en Internet». En Teatro e Internet en la primera década del siglo XXI, José Romera Castillo (ed.), 140-152. Madrid: Verbum.

OROZCO VERA, M. J. (2012). «Erotismo y espacios de tránsito bajo el prisma de la brevedad dramática: Día Mundial del Teatro. Sevilla 2010, espectáculo dirigido por Alfonso Zurro». En Erotismo y teatro en la primera década del siglo XXI, José Romera Castillo (ed.), 347-359. Madrid: Visor Libros.

ROMERA CASTILLO, J. (2008). «Hacia un estado de la cuestión sobre teatro y nuevas tecnologías en España». Signa. Revista de la Asociación de Semiótica 17, 17-28 (disponible también en http://www.cervantesvirtual.com/obra/hacia-un-estado-de-la-cuestin-sobre-teatro-y-nuevastecnologas-en-espaa-0/).

- (2010). «Literatura, teatro y nuevas tecnologías: investigaciones en el SELITEN@T (España)».Epos XXVI, 409-420 (también puede leerse en http://congresosdelalengua.es/valparaiso/ponencias/lengua_comunicacion/romera_jose.htm).

- (2011a). «Sobre teatro y nuevas tecnologías». En su obra Teatro español entre dos siglos a examen, 388-409. Madrid: Verbum.

- (2011b). «El Centro de Investigación de Semiótica Literaria, Teatral y Nuevas Tecnologías». En su obra Pautas para la investigación del teatro español y sus puestas en escena, 21-45. Madrid: UNED.

- (2013). «Sobre teatro e Internet en el SELITEN@T». En Teatro e Internet en la primera década del siglo XXI, José Romera Castillo (ed.), 11-32. Madrid: Verbum. 
ROMERA CASTILLO, J. (ed.) (2004). Teatro, prensa y nuevas tecnologías (1990- 2003). Madrid: Visor Libros.

- (2011). El teatro breve en los inicios del siglo XXI. Madrid: Visor Libros.

- (2013). Teatro e Internet en la primera década del siglo XXI. Madrid: Verbum.

ROMERA CASTILLO, J. et alii (eds.) (1997). Literatura y multimedia. Madrid: Visor Libros.

ROMERO LÓPEZ, D. (2008). «Presentación». Signa. Revista de la Asociación de Semiótica 17, 13-16 (también en http://www.cervantesvirtual. com/obra/signa- revista-de-la-asociacin-espaola-de-semitica-nm17-2008-estado-de-la-cuestin-sobre-teatro-y-nuevas-tecnologas-presentacin-0/).

Recibido el 5 de junio de 2013.

Aceptado el 19 de septiembre de 2013. 
